\title{
OPEN Exploration into biomarker potential of region-specific brain gene co-expression networks
}

\author{
Yuqing Hang ${ }^{1}$, Mohammed Aburidi ${ }^{2}$, Benafsh Husain ${ }^{2}$, Allison R. Hickman ${ }^{1}$,
} William L. Poehlman ${ }^{1} \&$ F. Alex Feltus ${ }^{1,2,3 凶}$

The human brain is a complex organ that consists of several regions each with a unique gene expression pattern. Our intent in this study was to construct a gene co-expression network (GCN) for the normal brain using RNA expression profiles from the Genotype-Tissue Expression (GTEx) project. The brain GCN contains gene correlation relationships that are broadly present in the brain or specific to thirteen brain regions, which we later combined into six overarching brain mini-GCNs based on the brain's structure. Using the expression profiles of brain region-specific GCN edges, we determined how well the brain region samples could be discriminated from each other, visually with t-SNE plots or quantitatively with the Gene Oracle deep learning classifier. Next, we tested these gene sets on their relevance to human tumors of brain and non-brain origin. Interestingly, we found that genes in the six brain mini-GCNs showed markedly higher mutation rates in tumors relative to matched sets of random genes. Further, we found that cortex genes subdivided Head and Neck Squamous Cell Carcinoma (HNSC) tumors and Pheochromocytoma and Paraganglioma (PCPG) tumors into distinct groups. The brain GCN and mini-GCNs are useful resources for the classification of brain regions and identification of biomarker genes for brain related phenotypes.

The human brain is a complex system encompassing countless cells that coalesce into hundreds of different regions and patterns of functional connectivity ${ }^{1}$. The coherence between brain regions results in canonical functions like vision, language, and memory ${ }^{2}$. The complexity of the brain is mainly due to the spatial and temporal alteration of large amounts of gene expression during developmental specification and maturation ${ }^{3}$. However, the region-specific description and control of gene expression patterns across the human brain has yet to be fully revealed.

Fortunately, recent high-resolution genome wide transcriptome profiling studies provide deeper insight into brain gene expression, especially in the context of disease-associated expression shifts in different brain regions. For example, Twine et al. ${ }^{4}$ studied transcriptome profiles from both healthy brains and brains from patients with Alzheimer's disease (AD). They found significant differences in gene expression levels, splicing isoforms, and alternative transcription start sites between healthy and $\mathrm{AD}$ brains. Another group created a gene expression model based on transcriptome datasets of the healthy human brain from the Allen Brain Atlas. This model can be used to identify potentially new candidate genes implicated in neurological diseases using machine learning ${ }^{5,6}$. Other studies include identifying gene expression patterns related to developmental origin of brain regions, brain functions, and brain-specific diseases like autism ${ }^{7-9}$. A brain transcriptome resource we leveraged in this study is from the Genotype-Tissue Expression (GTEx) project ${ }^{10}$ that characterized 54 tissues from 948 human donors. Thirteen of those tissue were from specific regions of the human brain.

With the increasing number and diversity of high-resolution human brain gene expression datasets, it is becoming easier to detect polygenic biomarker systems relevant to a specific medical condition or brain region. For example, using pairwise gene expression correlation tests across genes in a gene expression matrix (GEM), a gene co-expression network $(\mathrm{GCN})$ can be constructed and utilized to detect co-functional gene sets ${ }^{11-14}$. In a GCN, each node represents a gene or gene product, and two nodes are connected by an edge if they have a significant co-expression relationship. A group of highly-connected genes in a GCN have a higher likelihood of being functionally related relative to a group of poorly connected genes. We construct GCNs using software called Knowledge-Independent Network Construction (KINC), which employs Gaussian Mixture Models (GMMs)

${ }^{1}$ Department of Genetics and Biochemistry, Clemson University, Clemson 29634, USA. ${ }^{2 B i o m e d i c a l ~ D a t a ~ S c i e n c e ~}$ and Informatics Program, Clemson University, Clemson 29634, USA. ${ }^{3}$ Center for Human Genetics, Clemson University, Clemson 29634, USA. ${ }^{\circledR}$ email: ffeltus@clemson.edu 
to cluster samples before a pairwise correlation calculation ${ }^{12}$. KINC deconvolutes mixed-condition pairwise expression profiles into condition-specific edges that can be merged into a condition specific GCN.

Condition-specific GCNs encompass candidate biomarker systems relevant to that condition. The effectiveness of the biomarkers to discriminate conditions can be formally tested using machine learning and other classification techniques. For example, Gene Oracle is a software package that implements a deep learning model to classify biological samples using gene expression features as input ${ }^{15}$. In the Gene Oracle algorithm, expression profiles of candidate gene sets are tested for significant non-random classification potential of sample types (i.e. classification labels). The gene sets are then decomposed into the most discriminatory candidate biomarker gene sets. In this approach, the relevance of a biomarker system is formally quantified and refined to the core biomarker features.

In this study, our goal was to identify condition-specific GCNs for normal human brain regions. Towards this goal, we constructed a brain GCN using a GEM derived from 13 brain RNA-seq datasets obtained from the GTEx project ${ }^{10}$. We deconvoluted the brain edges into brain region mini-GCNs and characterized highly interconnected genes. We then used Gene Oracle to classify the input brain samples with these mini-GCNs to test their biomarker potential on normal brain regions. Finally, we tested if the brain region-specific genes tumor expression profiles were able to discriminate the brain from non-brain human tumors.

\section{Results}

Brain region-specific gene co-expression network (GCN) construction. We wanted to identify region-specific gene co-expression patterns in the brain using $\mathrm{KINC}^{13}$. To do this, we analyzed 1671 GTEx gene expression profiles from 56,202 genes across 13 different brain regions. Prior to GCN construction, the GTEx GEM was preprocessed by $\log 2$ transformation of the expression values, applying the Kolmogorov-Smirnov test to remove outlier distributions, and performing quantile normalization on the GEM. The full brain GCN contains 1691 nodes and 7812 edges (Supplemental Table 1; Fig. 1A-right panel). The GCN was further dissected into 183 linked community modules (LCMs) ${ }^{16}$.

We then performed sample label enrichment for all modules and edges in the brain GCN (Supplemental Tables 2 and 3). In the sample label enrichment for modules, we considered to use the p-value threshold of $1 \mathrm{E}-3$ for significantly enriched modules. Because modules contain different numbers of genes, there are very few modules that are enriched significantly in only one brain region. Thus, we calculated the sample label enrichment for each edge. The edges with a p-value less than $1 \mathrm{E}-10$ were considered to be significantly enriched because this p-value is close to maximizing the number of edges and nodes. The number of enriched edges for each specific region with the adjusted p-value less than $1 \mathrm{E}-3,1 \mathrm{E}-5,1 \mathrm{E}-15$, and $1 \mathrm{E}-20$ were also collected separately and the tSNE visualization was ran for each of those gene sets (Supplemental Table 4 and Supplemental Figure 1). One can see from the results that even though the threshold p-value of $1 \mathrm{E}-15$ had more edges and nodes in total, most regions had a decrease in the number of specific edges. For example, the number of edges and nodes in the basal ganglia, cerebellum, and spinal cord regions was lower when the threshold was $1 \mathrm{E}-15$ compared to a p-value threshold of $1 \mathrm{E}-10$. Exceptions to this included the cortex specific edges, which increased slightly in number, and the hypothalamus where the number of specific edges increased strikingly. Furthermore, the tSNE plots showed that the region-specific genes cannot separate samples very well for $\mathrm{p}$-value thresholds of $1 \mathrm{E}-3$ and $1 \mathrm{E}-5$, while they can for thresholds of $1 \mathrm{E}-10,1 \mathrm{E}-15$, and $1 \mathrm{E}-20$. Thus, we used a p-value of $1 \mathrm{E}-10$ as the threshold of significance for the sample label enrichment for edges.

The identified region-specific sub-GCNs with adjusted p-value less than $1 \mathrm{E}-10$ are shown in Fig. 1B-right panel for each region. Global attributes for both full brain GCN and region sub-GCNs are shown in Table 1. For example, the 160 brain caudate (basal ganglia) samples significantly contributed to 2076 edges $(\mathrm{p}<1 \mathrm{E}-10)$ that contained 690 nodes and connected 131 modules. These brain caudate (basal ganglia) nodes had high connectivity $(\mathrm{k}=6.02)$, and among the 690 nodes, 33,554 eQTLs were found in the GTEx database. The average gene expression values for enriched nodes $(\mu=3.89, \sigma=2.49)$ was higher than all GTEx gene expression values $(\mu=0.57, \sigma=3.34)$.

We counted the number of modules, edges, and eQTLs that were enriched for each brain region (Fig. 2). Most modules were enriched in multiple brain regions. However, there were three modules enriched in only one specific brain region, and one more module enriched in two brain regions. Similar to the modules, the majority of the edges were not enriched in one single region, with the exception of 434 that were present in only one brain region. Most eQTLs were found in only one brain region, and the number of eQTLs decreased when there were more shared regions, except for the number of eQTLs shared by all 13 brain regions. For each region's enriched edges, we also counted how many of them were associated with other regions (Supplemental Figure 2). Most edges were enriched in more than one brain region.

For the 434 region-specific edges, we identified edges that were unique for one region as shown in Table 2. For example, 139 nodes and 200 edges were found that are only enriched in brain caudate (basal ganglia) samples. Only 22 genes out of the 139 unique caudate genes contained eQTLs and 917 eQTLs were found in total. On average, each unique node had 41.68 eQTLs. Of the 13 brain regions, ten contained region-specific edges. According to the anatomy of the brain, we combined some of the region-specific edges together to form six region-specific edge lists. For example, the basal ganglia consists of the caudate, the nucleus accumbens, and the putamen. Therefore, we combined those region-specific edges together. The cerebellar and cerebellar hemisphere samples were taken from the same site in the brain, so we combined those two lists together. The hippocampus only contained two region-specific edges, therefore it was not large enough to construct its own sub-GCN. In total, six overarching region-specific edge lists were generated from the brain GCN. We used these new sets to construct sub-GCNs and visualize their gene expression patterns. 
A

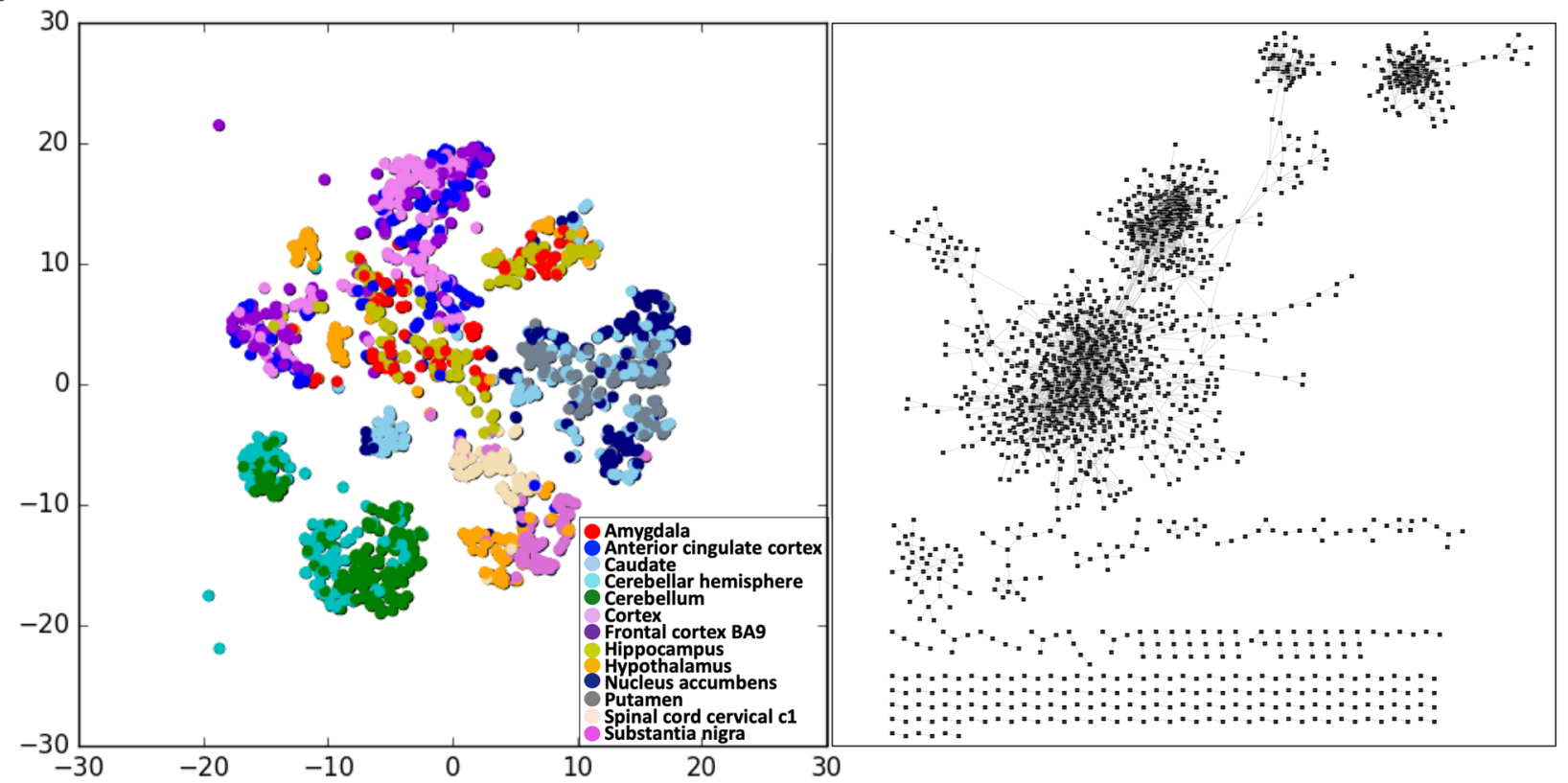

B
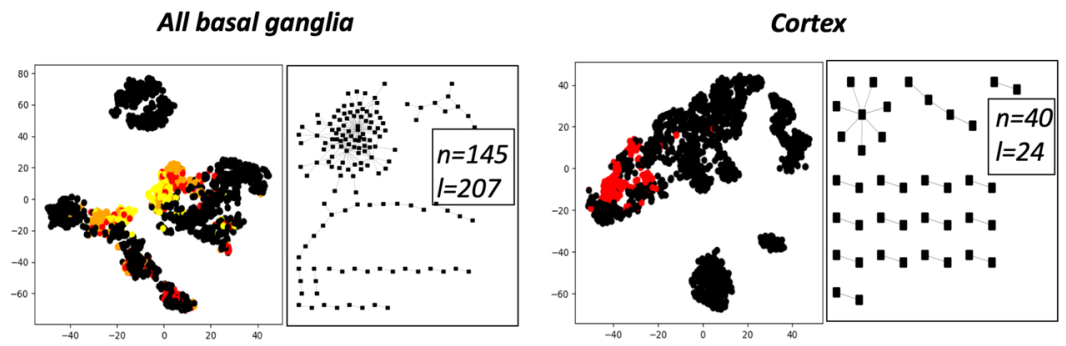

Cerebellum \& cerebellar hemisphere

Hypothalamus

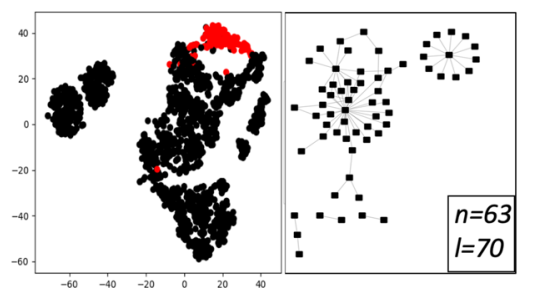

Substantia nigra

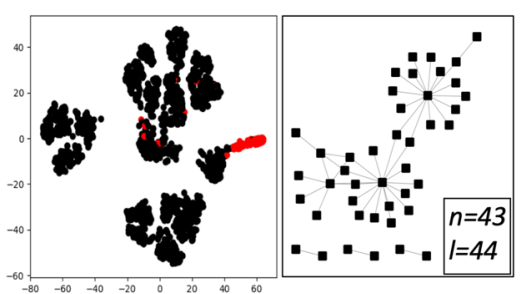

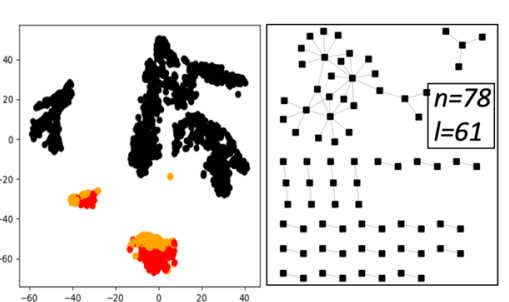

Spinal cord

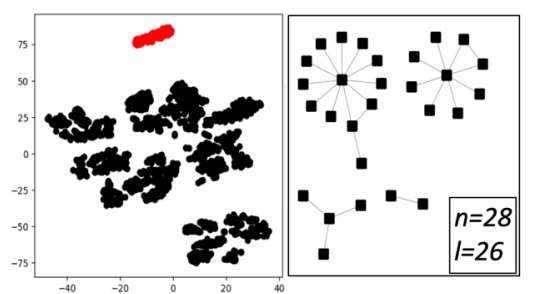

Figure 1. Normal brain gene co-expression network. (A) The right panel represents the whole gene co-expression network (GCN) constructed from 1671 GTEx brain RNAseq samples from 13 different brain regions. The left panel is the corresponding t-SNE visualization for the 1691 brain GCN genes where RNA expression profiles sorted regions into multiple clusters. Each color represents a different region shown in the legend. (B) Six brain region mini-GCNs are shown on the right side of each panel. Corresponding t-SNE visualization pictures for those region-specific genes are shown on the left side of each panel. Non-black dots in each $\mathrm{tSNE}$ plot represent the corresponding region-specific samples and black dots represent samples from all other regions. For all basal ganglia specific gene sets, red, orange and yellow dots represent caudate basal ganglia, nucleus accumben basal ganglia, and putamen basal ganglia samples respectively. The red and orange dots from cerebellum and cerebellar hemisphere specific gene sets represent cerebellum and cerebellar hemisphere samples respectively. All red dots from other region-specific gene sets represent the particular region-specific samples.

To see how region-specific gene subsets separate the brain regions, we performed t-SNE. t-SNE is a dimensionality reduction algorithm for visualization of high dimensional data into two or three dimensions ${ }^{17}$. Using all the genes from the full brain GCN as input to t-SNE, the regions separated at different degrees (Fig. 1A-left panel). The main observation was the separation of the cerebellum and cerebellar hemisphere samples from other region samples. The expression pattern for other brain regions mixed together and could not be distinguished.

Using different sets of region-specific sub-GCN genes as input to t-SNE, the region distribution varied (Fig. 1B-left panel). For example, the basal ganglia region, consisting of caudate basal ganglia, nucleus 


\begin{tabular}{|c|c|c|c|c|c|c|c|c|c|c|c|c|}
\hline Region & Samples & Nodes & Edges $^{\mathrm{a}}$ & Modules $^{\mathrm{a}}$ & $\begin{array}{l}\text { All genes } \\
\text { specific } \\
\text { eQTLs }\end{array}$ & $\begin{array}{l}{[\text { RNA] All }} \\
\text { genes mean }\end{array}$ & $\begin{array}{l}\text { [RNA] All } \\
\text { genes stdev }\end{array}$ & $\begin{array}{l}\text { [RNA] } \\
\text { Enriched } \\
\text { node mean }\end{array}$ & $\begin{array}{l}\text { [RNA] } \\
\text { Enriched } \\
\text { node stdev }\end{array}$ & $\mathbf{k}$ & Unique edges & $\begin{array}{l}\text { Unique } \\
\text { edge } \\
\text { percentage }\end{array}$ \\
\hline $\begin{array}{l}\text { Full network } \\
\text { (all regions) }\end{array}$ & 1671 & 1691 & 7812 & 183 & 38,549 & 0.57 & 3.34 & 3.65 & 2.71 & 9.24 & 434 & 0.06 \\
\hline $\begin{array}{l}\text { Brain amyg- } \\
\text { dala }\end{array}$ & 100 & 188 & 145 & 87 & 4,097 & 0.57 & 3.34 & 4.50 & 1.99 & 1.54 & 0 & 0.00 \\
\hline $\begin{array}{l}\text { Brain anterior } \\
\text { cingulate } \\
\text { cortex BA24 }\end{array}$ & 121 & 419 & 468 & 41 & 11,833 & 0.57 & 3.34 & 4.62 & 1.90 & 2.23 & 0 & 0.00 \\
\hline $\begin{array}{l}\text { Brain caudate } \\
\text { (basal ganglia) }\end{array}$ & 160 & 690 & 2076 & 131 & 33,554 & 0.57 & 3.34 & 3.89 & 2.49 & 6.02 & 200 & 0.10 \\
\hline $\begin{array}{l}\text { Brain cerebel- } \\
\text { lar hemisphere }\end{array}$ & 136 & 270 & 225 & 4 & 43,794 & 0.57 & 3.34 & 4.86 & 1.90 & 1.67 & 1 & 0.00 \\
\hline $\begin{array}{l}\text { Brain cerebel- } \\
\text { lum }\end{array}$ & 173 & 327 & 301 & 13 & 129,623 & 0.57 & 3.34 & 4.98 & 1.84 & 1.84 & 60 & 0.20 \\
\hline Brain cortex & 158 & 646 & 928 & 74 & 42,199 & 0.57 & 3.34 & 4.87 & 1.87 & 2.87 & 24 & 0.03 \\
\hline $\begin{array}{l}\text { Brain frontal } \\
\text { cortex BA9 }\end{array}$ & 129 & 545 & 735 & 54 & 20,048 & 0.57 & 3.34 & 5.03 & 1.81 & 2.70 & 0 & 0.00 \\
\hline $\begin{array}{l}\text { Brain hip- } \\
\text { pocampus }\end{array}$ & 123 & 377 & 909 & 114 & 8147 & 0.57 & 3.34 & 3.99 & 2.70 & 4.82 & 2 & 0.00 \\
\hline $\begin{array}{l}\text { Brain hypo- } \\
\text { thalamus }\end{array}$ & 121 & 440 & 1502 & 103 & 7853 & 0.57 & 3.34 & 3.67 & 2.59 & 6.83 & 70 & 0.05 \\
\hline $\begin{array}{l}\text { Brain nucleus } \\
\text { accumbens } \\
\text { (basal ganglia) }\end{array}$ & 147 & 536 & 693 & 73 & 24,106 & 0.57 & 3.34 & 4.77 & 1.83 & 2.59 & 5 & 0.01 \\
\hline $\begin{array}{l}\text { Brain puta- } \\
\text { men (basal } \\
\text { ganglia) }\end{array}$ & 124 & 427 & 466 & 75 & 14,446 & 0.57 & 3.34 & 4.70 & 1.84 & 2.18 & 2 & 0.00 \\
\hline $\begin{array}{l}\text { Brain spinal } \\
\text { cord cervi- } \\
\text { cal cl }\end{array}$ & 91 & 145 & 95 & 37 & 14,663 & 0.57 & 3.34 & 3.80 & 2.26 & 1.31 & 26 & 0.27 \\
\hline $\begin{array}{l}\text { Brain substan- } \\
\text { tia nigra }\end{array}$ & 88 & 111 & 84 & 51 & 2782 & 0.57 & 3.34 & 3.75 & 2.30 & 1.51 & 44 & 0.52 \\
\hline
\end{tabular}

Table 1. Normal brain GCN edge attributes. [1] For edge enrichment, we consider the significant edges for each sub-cluster as those with p-values less than 1E-10; [2] For module enrichment, we consider the significant modules for each sub-cluster will be those with p-values less than $1 \mathrm{E}-3$.

A

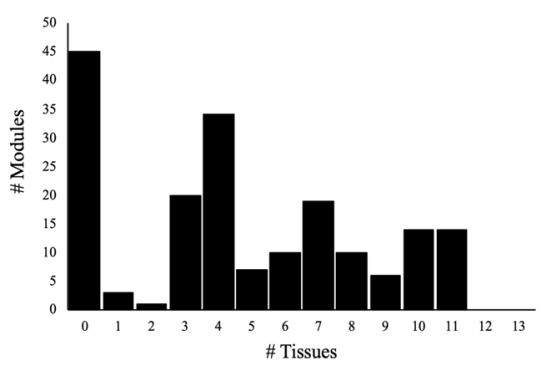

B

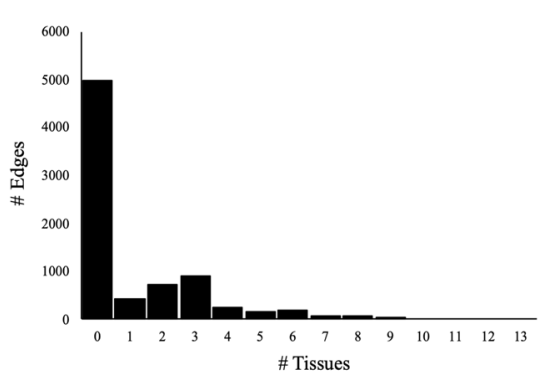

$\mathrm{C}$

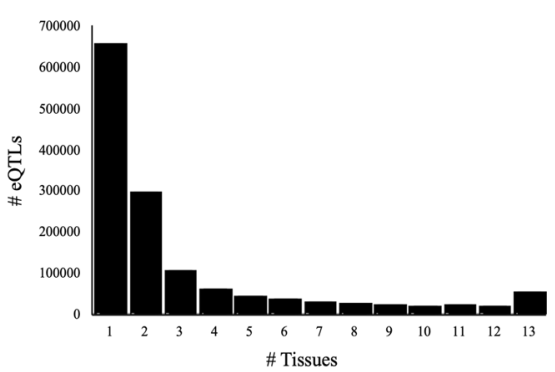

Figure 2. Brain region-specific GCN attributes. (A) Number of link community modules unique to $0-13$ brain regions. (B) Number of edges unique in 0-13 brain regions. (C) Number of region-specific edge associated GTEx eQTLs unique in 1-13 brain regions.

accumbens basal ganglia, and putamen basal ganglia samples, did not separate basal ganglia samples from other region samples based on the expression patterns of the 145 unique basal ganglia genes. This pattern was also observed in the cortex samples when using the 40 unique cortex genes as input. However, the expression pattern for 28 spinal cord unique nodes separated the spinal cord samples from any other brain region samples. Also, cerebellum specific genes were able to separate cerebellum and cerebellar hemisphere samples from all other samples. The t-SNE visualization for each region based on its enriched nodes is shown in Supplemental Figure 3.

We performed functional enrichment analysis on full brain GCN modules (Supplemental Table 5) as well as brain region-specific nodes (Supplemental Table 6). Table 3 lists the region-specific module information, and Table 4 lists their corresponding functional enrichment results.

To determine if the region-specific edges were coding or non-coding genes, we counted the gene classes for all GTEx input genes, brain GCN genes, as well as each region's specific gene list (Supplemental Table 7). About one 


\begin{tabular}{|l|r|r|r|l|l|}
\hline Region & Edges & Nodes & eQTLs & Nodes counted & eQTLs/node \\
\hline Full network (all regions) & 434 & 344 & 2228 & 59 & 37.76 \\
\hline Brain amygdala & 0 & 0 & 0 & 0 & 0 \\
\hline Brain anterior cingulate cortex BA24 & 0 & 0 & 0 & 0 & 0 \\
\hline Brain caudate (basal ganglia) & 200 & 139 & 917 & 22 & 41.68 \\
\hline Brain cerebellar hemisphere & 1 & 2 & 0 & 0 & 0 \\
\hline Brain cerebellum & 60 & 76 & 874 & 17 & 51.41 \\
\hline Brain cortex & 24 & 40 & 296 & 9 & 32.89 \\
\hline Brain frontal cortex BA9 & 0 & 0 & 0 & 0 & 0 \\
\hline Brain hippocampus & 2 & 4 & 0 & 0 & 0 \\
\hline Brain hypothalamus & 70 & 63 & 108 & 6 & 18 \\
\hline Brain nucleus accumbens (basal ganglia) & 5 & 9 & 23 & 1 & 23 \\
\hline Brain putamen (basal ganglia) & 2 & 4 & 0 & 0 & 0 \\
\hline Brain spinal cord cervical C1 & 26 & 28 & 0 & 0 & 0 \\
\hline Brain substantia nigra & 44 & 43 & 10 & 4 & 2.5 \\
\hline
\end{tabular}

Table 2. Unique region-specific edge attributes.

\begin{tabular}{|l|l|l|l|}
\hline Module & Edges & Enriched region & p-value \\
\hline M005 & 5 & Cerebellum & $4.20 \mathrm{E}-11$ \\
\hline M126 & 3 & Cerebellar hemisphere; cerebellum & $2.54 \mathrm{E}-78 ; 1.57 \mathrm{E}-102$ \\
\hline
\end{tabular}

Table 3. Region-specific module information.

\begin{tabular}{|c|c|c|c|c|}
\hline Module & Region & Adj. $\mathbf{p}^{1}$ & Term ID & Term definition \\
\hline M0005 & Cerebellum & $1.22 \mathrm{E}-03$ & MIM:114850 & CARBOXYPEPTIDASE A1 \\
\hline M0005 & Cerebellum & $1.22 \mathrm{E}-03$ & MIM:246600 & PANCREATIC LIPASE \\
\hline M0005 & Cerebellum & $1.22 \mathrm{E}-03$ & MIM:276000 & PROTEASE, SERINE, 1 \\
\hline M0005 & Cerebellum & $1.94 \mathrm{E}-03$ & GO:0005615 & Extracellular space \\
\hline M0005 & Cerebellum & $3.42 \mathrm{E}-03$ & GO:0006508 & Proteolysis \\
\hline M0005 & Cerebellum & $1.57 \mathrm{E}-03$ & GO:0008233 & Peptidase activity \\
\hline M0005 & Cerebellum & $5.63 \mathrm{E}-03$ & GO:0008236 & Serine-type peptidase activity \\
\hline M0005 & Cerebellum & $2.25 \mathrm{E}-03$ & GO:0016787 & Hydrolase activity \\
\hline M0005 & Cerebellum & $7.70 \mathrm{E}-03$ & GO:0061365 & Positive regulation of triglyceride lipase activity \\
\hline M0005 & Cerebellum & $9.55 \mathrm{E}-03$ & IPR001314 & Peptidase S1A, chymotrypsin family \\
\hline M0005 & Cerebellum & $8.05 \mathrm{E}-03$ & IPR018114 & Serine proteases, trypsin family, histidine active site \\
\hline M0005 & Cerebellum & $6.96 \mathrm{E}-03$ & IPR033116 & Serine proteases, trypsin family, serine active site \\
\hline M0005 & Cerebellum & $1.18 \mathrm{E}-03$ & PF00089 & Trypsin \\
\hline M0005 & Cerebellum & $9.42 \mathrm{E}-03$ & R-HSA-196854 & Metabolism of vitamins and cofactors \\
\hline M0126 & Cerebellar hemisphere; cerebellum & $2.18 \mathrm{E}-03$ & MIM:603140 & $\begin{array}{l}\text { PHOSPHATIDYLINOSITOL 5-PHOSPHATE 4-KINASE, } \\
\text { TYPE II, ALPHA }\end{array}$ \\
\hline M0126 & Cerebellar hemisphere; cerebellum & $2.18 \mathrm{E}-03$ & MIM:609410 & SYNAPTOJANIN 2 \\
\hline M0126 & Cerebellar hemisphere; cerebellum & $2.18 \mathrm{E}-03$ & MIM:610072 & ERMIN \\
\hline M0126 & Cerebellar hemisphere; cerebellum & $2.18 \mathrm{E}-03$ & MIM:616027 & ACTIN-BINDING PROTEIN ANILLIN \\
\hline M0126 & Cerebellar hemisphere; cerebellum & $8.74 \mathrm{E}-03$ & IPR031970 & Anillin, $\mathrm{N}$-terminal domain \\
\hline M0126 & Cerebellar hemisphere; cerebellum & $8.74 \mathrm{E}-03$ & IPR034973 & Synaptojanin-2, RNA recognition motif \\
\hline M0126 & Cerebellar hemisphere; cerebellum & $8.74 \mathrm{E}-03$ & IPR034974 & Synaptojanin-2 \\
\hline M0126 & Cerebellar hemisphere; cerebellum & $6.71 \mathrm{E}-03$ & PF08174 & Cell division protein anillin \\
\hline M0126 & Cerebellar hemisphere; cerebellum & $5.03 \mathrm{E}-03$ & PF08952 & Domain of unknown function (DUF1866) \\
\hline M0126 & Cerebellar hemisphere; cerebellum & $3.35 \mathrm{E}-03$ & PF16018 & Anillin N-terminus \\
\hline M0126 & Cerebellar hemisphere; cerebellum & $4.30 \mathrm{E}-03$ & R-HSA-1483255 & PI Metabolism \\
\hline M0126 & Cerebellar hemisphere; cerebellum & $1.73 \mathrm{E}-03$ & R-HSA-1660499 & Synthesis of PIPs at the plasma membrane \\
\hline
\end{tabular}

Table 4. Unique brain module functional enrichment analysis. [1] Bonferroni adjusted p-value $<0.01$. 
third of the GTEx input genes are protein coding genes, but of those from the brain GCN and the region-specific gene lists, almost all the genes are protein coding. Interestingly, among the 1,671 genes from brain GCN, there are 39 genes that are lncRNA (Supplemental Table 8). This could be our future topic of study.

Brain region biomarker validation. The six brain region-specific gene sets identified by GCN analysis were evaluated using Gene Oracle software which determines classification accuracy of a set of target genes relative to an identical number of random genes. We ran Gene Oracle phase I over 1671 samples from 13 different brain regions. Genes of each region's specific set were used as features to classify samples into 13 brain regions. The output accuracy of each region's specific set is shown in Fig. 3A. The following five sets showed significant classification potential: substantia nigra, spinal cord, hypothalamus, cortex, and cerebellum. Surprisingly, the sixth set (basal ganglia) was not significant as random genes provided a similar accuracy. To show the precise classification and the contribution of a region-specific gene set to each class, we generated a confusion matrix for each set (Fig. 3B). Darker green correlates to a higher classification accuracy. We observed that in most cases the region-specific gene sets classified their respective regions more accurately than other sets.

The three smallest significant sets of these five sets were selected for full combinatorial analysis with phase II of Gene Oracle. These included the spinal cord, cortex, and substantia nigra gene sets. Gene Oracle identified the genes which contributed the most to overall classification accuracy (candidate genes) for each of these region-specific sets. Figure 4 contains heatmaps which show the normalized frequency of a gene in a subset at a given iteration of the combinatorial analysis for the spinal cord, cortex and substantia nigra sets. The first three rows of the heatmaps had constant frequency values because all possible combinations of genes were evaluated, hence all genes appeared equivalently in the first three iterations. For the rest of the iterations, the distribution of the frequencies became varied and the most frequent genes appeared. Using the heatmaps, we determined the candidate genes of the three sets to be those with an aggregate frequency of at least one-half the standard deviation above the mean. The rest of the genes were considered "non-candidate" genes. Table 5 shows the candidate genes identified by Gene Oracle for each of these three regions.

Due to computational constraints imposed by the large number of genes, we used a Random Forest approach in lieu of Gene Oracle phase 2 for the hypothalamus and cerebellum sets. We used feature_importances_() built in function to output the most important features (i.e. genes), which were then considered candidates for these two regions. To compare to Gene Oracle, we also ran Random Forest to identify the candidate genes for spinal cord, cortex and substantia nigra. Candidate genes identified by Random Forest are shown in Table 6. The genes denoted in bold are common between the two methods.

To verify the classification potential for the candidate genes, we ran the Random Forest again for each candidate set shown in Tables 5 and 6 . Figure 5 shows, for each region-specific set, the classification accuracy of (1) the original region-specific set, (2) the candidate set identified using Gene Oracle, (3) the non-candidate set identified using Gene Oracle, and to compare to Random Forest, (4) the candidate set identified using Random Forest, (5) the non-candidate set identified using Random Forest. Additionally, the accuracy of each category was compared with the averaged accuracy of 50 random sets of equal size of genes of each set. In all cases, the difference in accuracy from random is highest in the candidate set and the lowest in the non-candidate sets. Furthermore, the candidate set identified by Gene Oracle exhibits a higher difference than those identified by Random Forest as shown in Fig. 5A.

Brain region biomarker potential for human brain tumors. We were interested to see how the brain region-specific genes could separate abnormal brain samples. For each region-specific gene set, we ran t-SNE on 1,431 tumor samples with four tumor types from The Cancer Genome Atlas (TCGA) as seen in Fig. 6. The tumor types were glioblastoma multiforme (GBM), lower grade glioma (LGG), head and neck squamous cell carcinoma (HNSC), and pheochromocytoma and paraganglioma (PCPG). Most of the region-specific genes could separate HNSC and PCPG tumors apart, while LGG and GBM samples could not be separated. The t-SNE visualization based on 40 cortex specific genes separated HNSC samples and PCPG samples into different subgroups. For each region-specific tSNE plot, the brain tumors (both LGG and GBM) were separated into 2-3 subgroups. The t-SNE visualizations of four TCGA subtypes based on enriched nodes for all 13 regions are shown in Supplemental Figure 4. We also ran t-SNE of the 40 cortex specific genes on TCGA tumor data for gender, race and stage. None of these factors could separate tumor clusters (Supplemental Figure 5). tSNE visualization on only brain tumors with IDH mutation annotation is shown in Supplemental Figure 6. For whole brain GCN genes, LGG and GBM were separated apart, but some LGG samples were more similar to GBM samples. The IDH mutant samples were more clearly separated from non-IDH mutant samples. LGG and GBM samples could not be separated using region-specific genes, while IDH mutated samples could be separated with non-IDH mutated samples. IDH mutated samples were also divided into several subgroups using each region's specific gene list. For example, for tSNE based on substantia nigra specific genes, almost all of the upper samples contain an IDH mutation, while almost all of the bottom samples did not contain an IDH mutation.

In order to see if the brain region-specific genes were important in different tumor types, we aggregated the mutation rates of five TCGA tumor types [GBM, LGG, HNSC, PCPG, and kidney renal clear cell carcinoma (KIRC)] for the six region-specific gene sets and one kidney gene set which contains the 20 most mutated genes in KIRC and their corresponding randomized control genes. As shown in Table 7, the number of mutated genes for all seven gene sets in GBM, LGG and HNSC was significantly higher than that for their corresponding random sets ( $p$-value $<0.01$ ). However, the number of mutated sub-brain specific genes was not significantly higher than the random sets in PCPG tumor. In KIRC tumors, only cerebellum specific gene set were significantly higher in mutated genes than randomly mutated genes. None of the other five region-specific gene sets had significantly numbers of higher mutated genes relative to a similar number of random genes. 
A

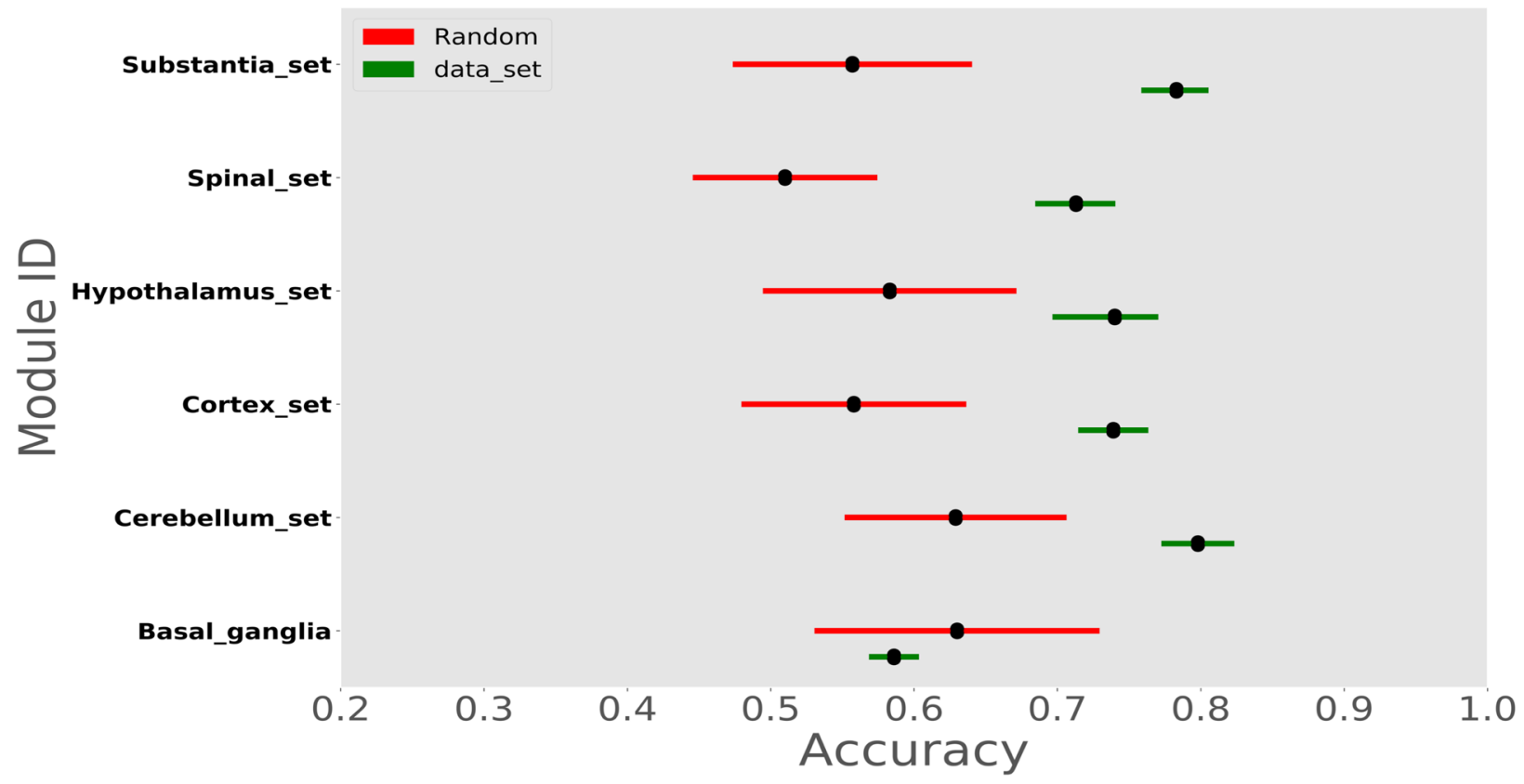

B

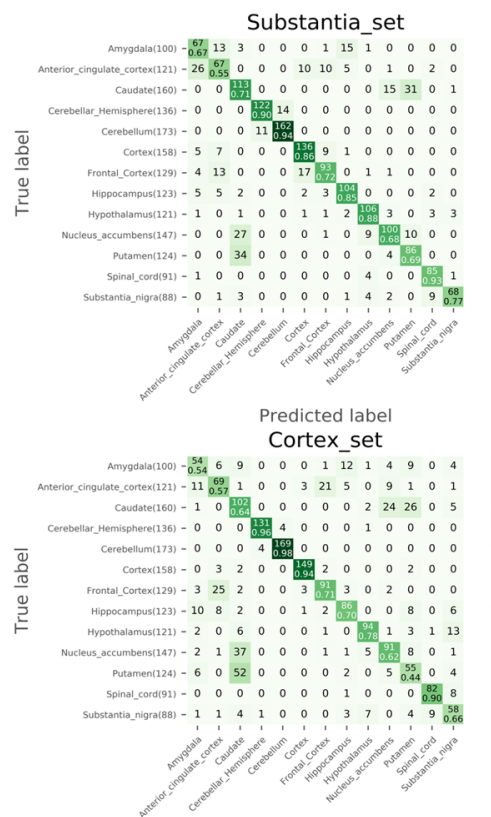

Predicted label

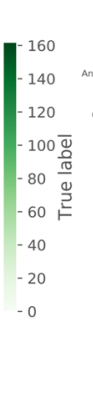

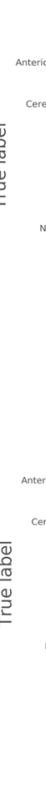

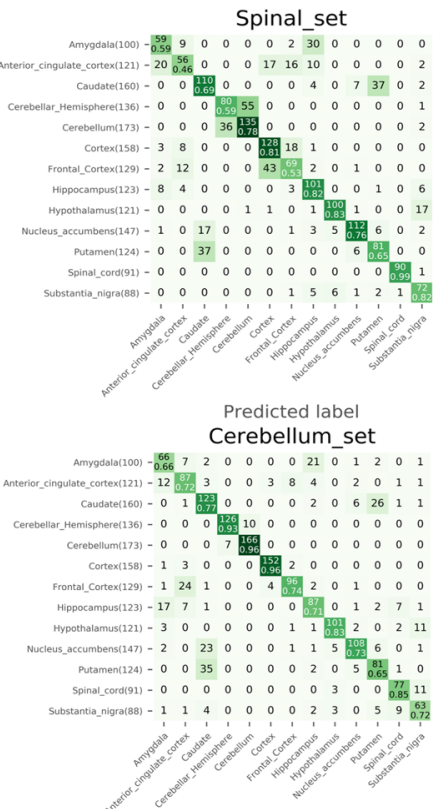

Predicted label

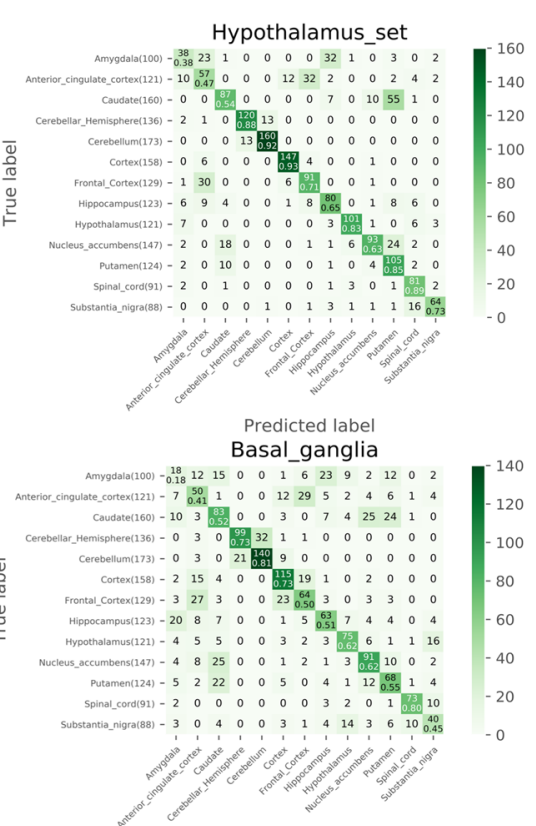

Predicted label

Figure 3. Gene Oracle classification of brain regions with brain region-specific edges. (A) Classification accuracy (X-axis) of region-specific gene sets (Y-axis; green bars) versus matched number of random genes (red bars) over 1671 GTEx brain samples from 13 different brain regions. (B) Confusion plot showing precise classifications (diagonal boxes) and misclassified samples for each region-specific gene sets. The upper number in the diagonal boxes indicates the number of samples that are correctly classified, and the lower number indicates its percent for each class. Other boxes show a number of misclassified samples.

\section{Discussion}

In this study, we constructed a normal brain GCN and identified edges that were specific to 13 brain regions. 

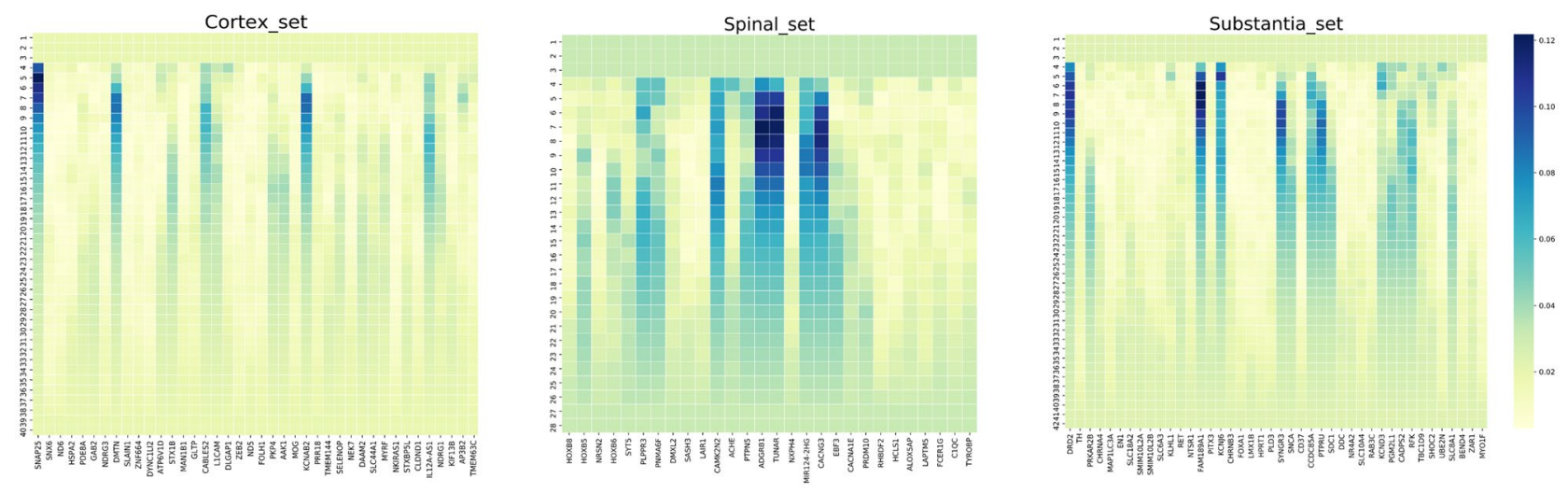

Figure 4. Combinatorial analysis of spinal cord, cortex and substantia nigra gene sets. Heatmaps depicting the frequency of genes present in the classification subsets that were generated at each Gene Oracle Phase 2 iteration. Each row is an iteration and each column is a gene from the cortex/spinal/substantia nigra sets. Darker colors correspond to higher frequencies.

\begin{tabular}{|l|l|}
\hline Region-specific set & Candidate genes identified by Gene Oracle phase II \\
\hline Substantia nigra & DRD2, FAM189A1, KCNJ6, SYNGR3, SNCA, CCDC85A, PTPRU, KCND3, CADPS2, RFK, SLC8A1 \\
\hline Cortex & SNAP25, DMTN, STX1B, CABLES2, L1CAM, PKP4, AAK1, KCNAB2, DAAM2, IL12A-AS1 \\
\hline Spinal cord & PLPPR3, CAMK2N2, PTPN5, ADGRB1, TUNAR, MIR124-2HG, CACNG3 \\
\hline
\end{tabular}

Table 5. Gene oracle candidate genes for brain GTEx dataset.

\begin{tabular}{|l|l|}
\hline Region-specific set & Candidate genes identified by Random Forest \\
\hline Substantia nigra & DRD2, TH, EN1, KLHL1, RET, KCNJ6, CHRNB3, SDC1, DDC, CADPS2, TBC1D9 \\
\hline Cortex & SNAP25, CABLES2, L1CAM, DLGAP1, PKP4, KCNAB2, NKIRAS1, STXBP5L, IL12A-AS1, TMEM63C \\
\hline Spinal cord & PNMA6F, CAMK2N2, PTPN5, TUNAR, NXPH4, MIR124-2HG, EBF3 \\
\hline
\end{tabular}

Table 6. Random Forest candidate genes for brain GTEx dataset. Genes in bold emphasis are common between the two methods.

After merging edges from similar regions, six brain region-specific GCNs were identified. Functional enrichment for both region-specific modules and the six sub-GCNs provided evidence that these genes encode brain functions. For example, as shown in tissue specific genes functional enrichment analysis in Supplemental Table 6, several edges specific for the substantia nigra were associated with the production of dopamine and other neurotransmitters, as well as their response to amphetamine and nicotine. In addition, both the hypothalamus and basal ganglia specific gene sets are enriched for cilium related functions, such as cilium, cilium movement, motile cilium, and cilium assembly. Cilia play an important role in modulating neurogenesis, cell polarity, axonal guidance and possibly adult neuronal function, which is related to brain development ${ }^{18}$. As expected, the brain GCN encodes brain function.

A prime motivation of our study was to test our approach to identify brain biomarker systems that can distinguish brain regions based upon region-specific co-expression relationships. The idea was that co-expressed genes unique to a brain region would be better biomarkers for sorting samples into normal and aberrant states that involve that region of the brain. Using t-SNE, we visualized the brain region clustering potential of these gene sets. Some gene sets separated regions well (e.g. spinal cord genes, substantia nigra genes, and cerebellum and cerebellar hemisphere genes), while others could not separate samples from each brain region. These visual results suggested that the biomarker sets have varied discriminatory potential.

The six brain region-specific gene sets were also evaluated for quantitative classification potential using a deep learning approach implemented in Gene Oracle to both classify samples from 13 brain regions and identify core candidate gene subsets which play the most important role in brain region classification. Using phase I of Gene Oracle, we examined the classification potential of six gene sets. All sets, except the basal ganglia set, showed significant mean classification accuracy relative to the mean accuracy of the same size of random gene sets (Fig. 3A). The confusion matrix of the basal ganglia gene set, which showed the precise classification and the contribution of each set to each region classification in Fig. 3B, possibly explains why the basal ganglia specific gene set had low classification accuracy. Because the caudate, nucleus accumbens and putamen all belong to the basal ganglia, they are physically located very close to each other. The combined basal ganglia gene set consisted of genes that were only enriched for one of these three regions. However, when we ran Gene Oracle 


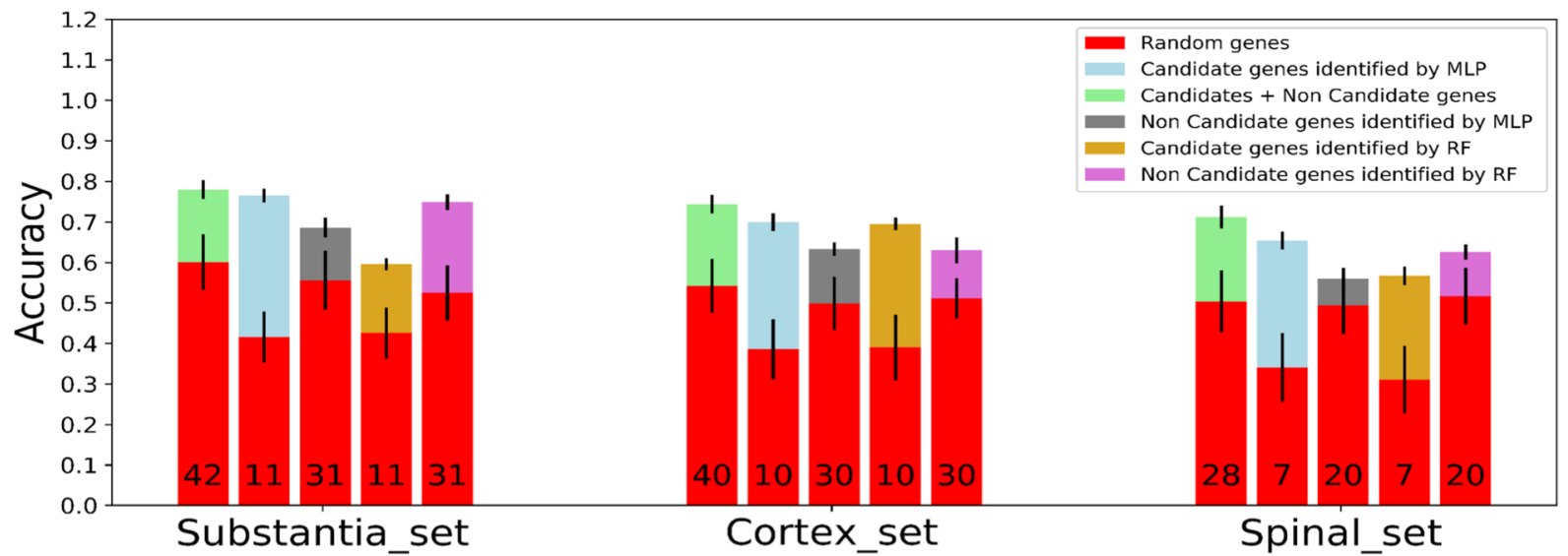

B

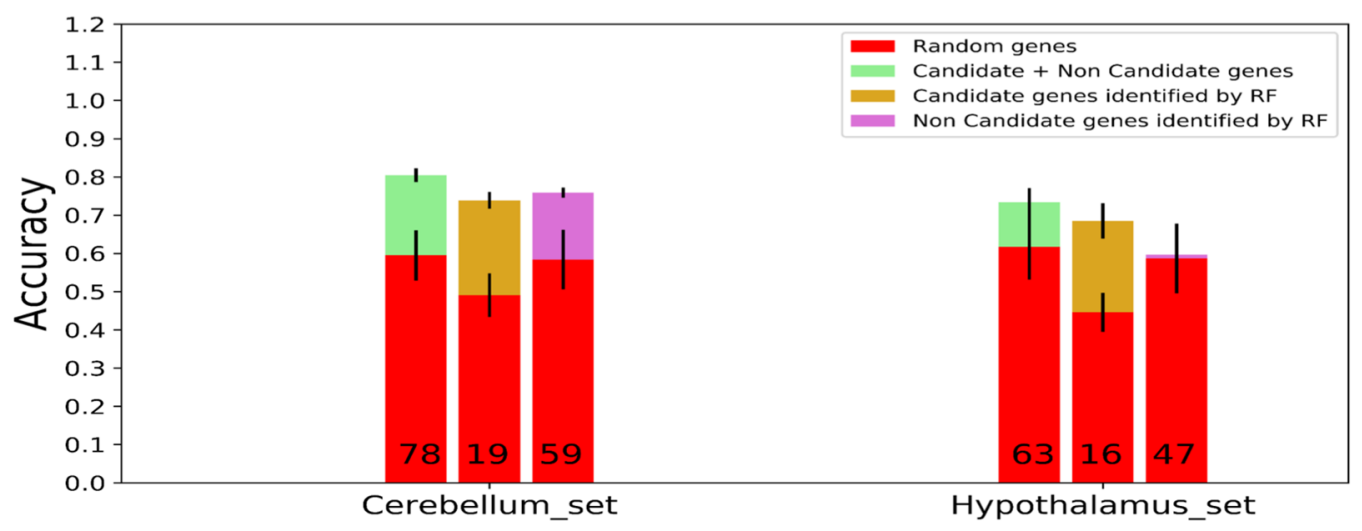

Figure 5. Classification potential for decomposed gene sets. (A) Classification accuracies for the full regionspecific gene sets (green) were compared to accuracies of the candidate genes identified by Gene Oracle (blue), non-candidate genes identified by Gene Oracle (gray), candidate genes identified by Random Forest (orange), and non-candidate genes identified by Random forest (purple). (B) Same as (A) but only for decomposed genes identified by Random Forest.

classification, we did not combine the above three regions together. Thus, for example, it is possible that the basal ganglia specific gene sets misclassified caudate into nucleus accumbens or putamen. The same explanation can be applied to the case that most of the sets show a high percentage of misclassification between cerebellar hemisphere and cerebellum. From the confusion plots, we can tell that similar brain regions had the trend to be misclassified with each other, which decreased the classification accuracy. Interestingly, some region-specific gene sets showed the ability to more accurately classify their regions. For example, when the genes of the spinal cord set were used as features, the model was able to classify the spinal cord samples with a $99 \%$ accuracy, which is higher when compared to other regions. These results reflect the fact that the region-specific genes can hold a higher predictive power for that region.

We used condition-specific GCN analysis via KINC to identify biomarker candidates. We were able to go one step further using Gene Oracle phase II and Random Forest feature extraction algorithms to identify genes which contributed the most to the overall classification accuracy (candidate genes) for each of the smallest three region-specific sets (substantia nigra, cortex and spinal cord sets). Once compared to the important genes identified by Random Forest, Gene Oracle showed a higher accuracy and a larger increase in accuracy when these genes were used as features (Fig. 5A). For example, the blue box represents the accuracy increase once candidate genes of substantia nigra, cortex, and spinal cord candidate gene sets were used as feature inputs to Gene Oracle. This represents a much higher accuracy compared to the random set (red) and the set identified through the use of Random Forest as a classifier model (orange). More interestingly, Gene Oracle provided deeper resolution than t-SNE. The left panels of Fig. 1B illustrate the large overlap between brain regions whereas Gene Oracle was able to easily discriminate the regions with high accuracy compared to random gene sets. The confusion matrices support this point as well, see Fig. 3B. These results demonstrate the power in utilizing deep learning technology for biomarker gene discovery.

After characterization of the biomarker potential of the brain genes on normal GTEx brain regions, we wanted to test the biomarker systems for classification potential of aberrant brain tissue. For this test, we chose LGG and GBM brain tumors as well as tumors that originated from other organs. t-SNE visualization was performed using TCGA tumor RNAseq expression profiles for the brain genes on samples from four different tumor types. Most 
Basal ganglia (145 genes)

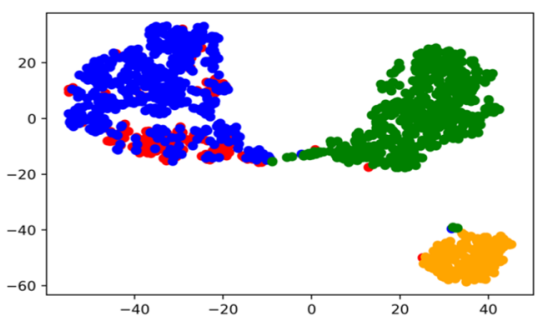

Hypothalamus (63 genes)

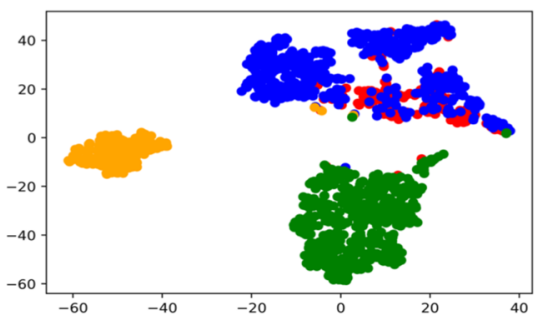

Cerebellum (76 genes)

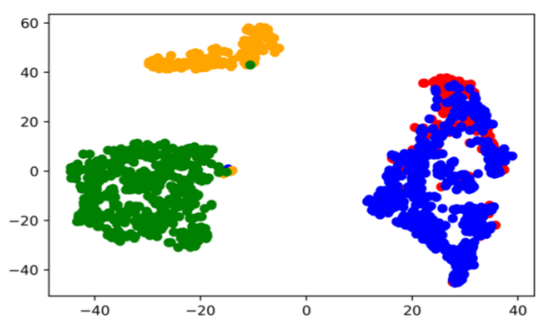

Spinal cord (28 genes)

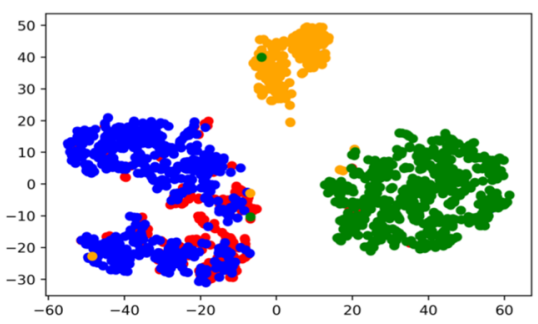

Cortex (40 genes)

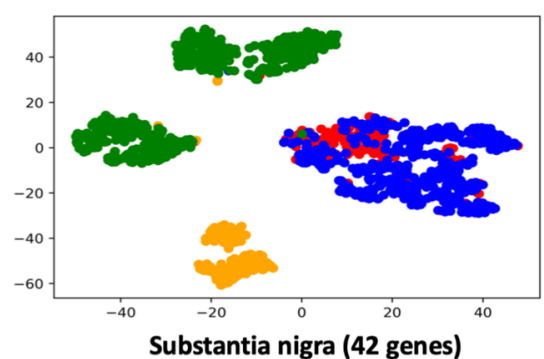

Substantia nigra (42 genes)

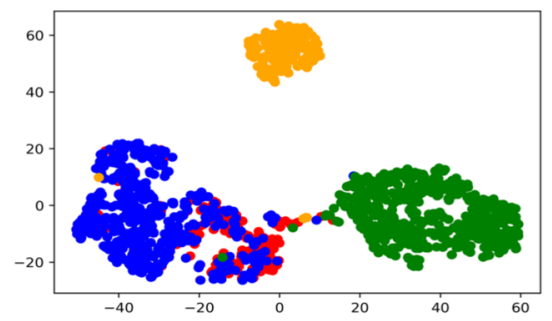

\begin{tabular}{lllllll}
\hline GBM & $\bullet$ & HNSC & $\bullet$ & LGG & PCPG \\
\hline
\end{tabular}

Figure 6. $t-S N E$ visualization of region-specific genes on TCGA tumor data. $t$-SNE was performed using TCGA RNAseq data from brain region sub-GCN genes. 1431 tumor samples from four tumor subtypes are shown. Tumor RNA expression profiles sorted regions into multiple clusters. Each color represents different regions. Red represents GBM; green represents HNSC; blue represents LGG; yellow represents PCPG.

of the region-specific genes could separate HNSC and PCPG tumors while LGG and GBM samples could not be separated. Interestingly, even though LGG and GBM samples could not be separated apart, those tumor samples can still be separated into several subgroups. This is partially due to the status of the IDH1/2 mutation which is very common in $\mathrm{LGG}^{19}$ (Supplemental Figure 6). The IDH mutant gliomas can be further divided into smaller sub-groups as well. Moreover, the t-SNE plots showed that the 40 cortex specific genes separated HNSC samples and PCPG samples into different sub-groups. As with the normal brain samples, there was mixed potential of the genes to sort human tumors.

Interestingly, we found that many of the brain region-specific GCN edges were mutated in tumors. As shown in Table 7, the number of mutated genes in all six brain region-specific gene sets was significantly higher than the number of mutated genes in the list of size-controlled random genes for GBM, LGG, and HNSC (p-value less than 0.01), but not for PCPG and KIRC. GBM and LGG represent tumors that originate in the brain. HNSC is not a brain cancer, but it originates in the squamous cells that line the moist, mucosal surfaces inside the head and neck, such as mouth, nose, throat, larynx, sinuses, or salivary glands ${ }^{20}$. PCPG originates mainly on the adrenal gland and only a few cases of paraganglioma localize in the neck and head ${ }^{21}$. KIRC originates from the kidney. The brain region-specific gene sets had a higher mutation rate in the brain tumors (LGG and GBM) than other tumors when compared to random genes. Interestingly, all brain region-specific gene sets had significantly higher mutation rate than random genes in HNSC, which indicates that these six brain specific gene sets could be important in HNSC tumor formation. Furthermore, cerebellum specific gene sets showed significantly higher mutation rate for KIRC, which means these 78 cerebellum specific genes may also play an important role in KIRC formation and development. The kidney specific gene set had higher mutation rates in almost all five listed tumor types. This might be because we chose the top 20 most mutated genes in KIRC as identified in the TCGA data portal. The 20 chosen genes are not necessarily specific to kidney tumors, and therefore could also be highly mutated in other tumors. Thus, these genes that are mutated in KIRC also have significantly high mutation rates in HNSC, LGG and PCPG.

In conclusion, this study describes how condition-specific candidate biomarker systems can be discovered using GCN analysis and we describe how machine learning approaches can be used to measure the quality of the biomarker sets. Further, we believe that the significant condition-specific relationships are worthy of deeper analysis into why they are present in specific brain regions. In the future we intend to further investigate the biological significance of these edges, including an examination of normal region eQTL regulation of these gene datasets to find important transcription factors and binding sites that may become altered during tumorigenesis.

\section{Methods}

Input data and gene expression matrix (GEM) preparation. All available gene-level TPM (transcripts per million) files for 13 normal brain region samples were downloaded from the Genotype-Tissue Expression (GTEx) project version $7^{10}$. 1671 samples were downloaded-each containing measurements of 56,202 genesand merged into a GEM. The matrix underwent preprocessing steps, including log base 2 transformation, quality control, and quantile normalization, using the preprocessCore R library ${ }^{22}$. The Kolmogorov-Smirnov test (KS 


\begin{tabular}{|c|c|c|c|c|c|c|c|c|c|c|c|c|}
\hline \multirow[b]{3}{*}{ Region } & TS & Polymorphism & Tumor & Mutated & Mutated & & TS genes & Random genes & & \begin{tabular}{|l|} 
TS genes \\
\end{tabular} & Random genes & \\
\hline & Genes & $\begin{array}{l}\text { Detection } \\
\text { method }\end{array}$ & Type & TS genes & Randomized & P-value & Mutated & Mutated & P-value & Total & Total & P-value \\
\hline & & & & & $\begin{array}{l}\text { Control gene } \\
\text { mean }\end{array}$ & & Tumors & Tumors mean & & Mutations & $\begin{array}{l}\text { Mutations } \\
\text { mean }\end{array}$ & \\
\hline Basal ganglia & 145 & Muse & GBM & 122 & 77.1 & $<0.01$ & 139 & 114.77 & 0.12 & 446 & 308.3 & 0.04 \\
\hline Basal ganglia & 145 & Muse & HNSC & 124 & 84.2 & $<0.01$ & 286 & 248.61 & 0.05 & 610 & 496.47 & 0.09 \\
\hline Basal ganglia & 145 & Muse & LGG & 96 & 63.6 & $<0.01$ & 106 & 79.55 & 0.08 & 389 & 316.2 & 0.1 \\
\hline Basal ganglia & 145 & Muse & PCPG & 10 & 8.7 & 0.23 & 11 & 9.53 & 0.23 & 12 & 9.89 & 0.21 \\
\hline Basal ganglia & 145 & Muse & KIRC & 67 & 55.5 & 0.03 & 118 & 91.4 & 0.04 & 150 & 115.49 & 0.05 \\
\hline Cerebellum & 78 & Muse & GBM & 68 & 44.9 & $<0.01$ & 154 & 86.21 & 0.01 & 402 & 207.68 & 0 \\
\hline Cerebellum & 78 & Muse & HNSC & 69 & 48.4 & $<0.01$ & 293 & 196.33 & 0 & 703 & 344.58 & 0 \\
\hline Cerebellum & 78 & Muse & LGG & 60 & 37.8 & $<0.01$ & 100 & 53.93 & 0.02 & 428 & 202.14 & 0 \\
\hline Cerebellum & 78 & Muse & PCPG & 9 & 5.48 & 0.05 & 10 & 6.13 & 0.06 & 10 & 6.36 & 0.07 \\
\hline Cerebellum & 78 & Muse & KIRC & 53 & 33.39 & $<0.01$ & 102 & 62.69 & 0 & 137 & 73.17 & 0 \\
\hline Cortex & 40 & Muse & GBM & 34 & 23.5 & $<0.01$ & 49 & 45.57 & 0.32 & 118 & 103.52 & 0.26 \\
\hline Cortex & 40 & Muse & HNSC & 36 & 25.4 & $<0.01$ & 126 & 122.54 & 0.35 & 194 & 176.84 & 0.26 \\
\hline Cortex & 40 & Muse & LGG & 27 & 19.4 & $<0.01$ & 36 & 27.44 & 0.17 & 129 & 103.29 & 0.18 \\
\hline Cortex & 40 & Muse & PCPG & 4 & 2.45 & 0.1 & 4 & 2.91 & 0.19 & 4 & 2.99 & 0.2 \\
\hline Cortex & 40 & Muse & KIRC & 19 & 16.95 & 0.18 & 35 & 34.35 & 0.36 & 39 & 37.99 & 0.38 \\
\hline Hypothalamus & 63 & Muse & GBM & 60 & 33 & $<0.01$ & 92 & 54.57 & 0.04 & 196 & 125.23 & 0.04 \\
\hline Hypothalamus & 63 & Muse & HNSC & 54 & 36.5 & $<0.01$ & 175 & 135.66 & 0.04 & 298 & 202.4 & 0.03 \\
\hline Hypothalamus & 63 & Muse & LGG & 44 & 27.4 & $<0.01$ & 56 & 37.39 & 0.04 & 188 & 134.15 & 0.06 \\
\hline Hypothalamus & 63 & Muse & PCPG & 2 & 3.6 & 0.75 & 2 & 3.95 & 0.78 & 2 & 4.14 & 0.78 \\
\hline Hypothalamus & 63 & Muse & KIRC & 28 & 22.54 & 0.01 & 48 & 41.54 & 0.17 & 55 & 46.45 & 0.16 \\
\hline Spinal cord & 28 & Muse & GBM & 20 & 12.3 & $<0.01$ & 33 & 20.4 & 0.05 & 74 & 41.44 & 0.02 \\
\hline Spinal cord & 28 & Muse & HNSC & 22 & 13.5 & $<0.01$ & 86 & 57.66 & 0.05 & 113 & 70.03 & 0.04 \\
\hline Spinal cord & 28 & Muse & LGG & 21 & 9.9 & $<0.01$ & 22 & 12.48 & 0.04 & 77 & 42.4 & 0.02 \\
\hline Spinal cord & 28 & Muse & PCPG & 1 & 1.1 & 0.3 & 1 & 1.2 & 0.34 & 1 & 1.2 & 0.34 \\
\hline \begin{tabular}{l|l} 
Spinal cord \\
\end{tabular} & 28 & Muse & KIRC & 11 & 8.21 & 0.12 & 19 & 15.2 & 0.23 & 21 & 16.01 & 0.17 \\
\hline Substantia nigra & 43 & Muse & GBM & 33 & 23.4 & $<0.01$ & 61 & 45.25 & 0.11 & 123 & 98.89 & 0.15 \\
\hline Substantia nigra & 43 & Muse & HNSC & 36 & 25.4 & $<0.01$ & 143 & 114.3 & 0.1 & 198 & 162.79 & 0.14 \\
\hline Substantia nigra & 43 & Muse & LGG & 27 & 19.9 & $<0.01$ & 41 & 28.45 & 0.05 & 139 & 101.72 & 0.08 \\
\hline Substantia nigra & 43 & Muse & PCPG & 2 & 2.74 & 0.5 & 7 & 3.2 & 0.05 & 8 & 3.28 & 0.03 \\
\hline Substantia nigra & 43 & Muse & KIRC & 20 & 16.76 & 0.13 & 30 & 33.29 & 0.59 & 30 & 36.66 & 0.7 \\
\hline \begin{tabular}{l|l} 
Kidney & \\
\end{tabular} & 20 & Muse & GBM & 19 & 13.44 & 0.01 & 18 & 32.43 & 0 & 34 & 67.57 & 0 \\
\hline Kidney & 20 & Muse & HNSC & 20 & 14.07 & $<0.01$ & 56 & 90.82 & 0 & 62 & 121.02 & 0 \\
\hline Kidney & 20 & Muse & LGG & 19 & 11.43 & $<0.01$ & 8 & 18.48 & 0 & 44 & 65.24 & 0 \\
\hline Kidney & 20 & Muse & PCPG & 12 & 1.8 & $<0.01$ & 1 & 1.96 & 0 & 1 & 1.98 & 0 \\
\hline Kidney & 20 & Muse & KIRC & 19 & 10.55 & $<0.01$ & 256 & 25.96 & 0 & 507 & 30.32 & 0 \\
\hline
\end{tabular}

Table 7. Mutation rates for brain region-specific gene sets in five TCGA tumors.

Dval $>0.15)^{23}$ was performed to test for outlier samples. No samples qualified as an outlier, so we continued with a quantile normalization of the matrix to reduce any technical noise. All FPKM (fragments per kilobase of gene per million read pairs) files for GBM, LGG, HNSC, and PCPG patients were downloaded from TCGA using the GDC Data Transfer Tool ${ }^{24}$. 1431 samples with 60,483 genes were aggregated into a GEM. The GEM underwent the same preprocessing steps as the GTEx GEM.

Gene co-expression network construction. KINC (https://github.com/SystemsGenetics/KINC) was used to identify gene correlation relationships within the normalized GTEx brain GEM. The algorithm calculates correlation for each gene pair after clustering samples using GMMs. Only clusters with equal to or more than 30 samples underwent Spearman correlation. We submitted 50,000 KINC similarity jobs on the Open Science $\mathrm{Grid}^{25}$ by using the OSG-KINC similarity workflow ${ }^{26}$. The workflow was accomplished using the Pegasus Workflow manager ${ }^{27}$. Normalized TPM expression values less than 0 were ignored. KINC similarity output was transferred to Clemson University's Palmetto Cluster via Globus. The KINC significance threshold of 0.8961 was found by using a random matrix thresholding (RMT) algorithm within the KINC thresholding script. The GTEx brain GCN was then constructed by extracting all edges with correlations $>0.8961$ using the KINC extract script. 183 linked community modules (LCMs) were identified by the linkcomm R packages with a minimum cluster size of 3 edges $^{16}$. The full GCN is shown in Supplemental Table 1. 
Edge and module sample enrichment analysis. All identified modules and edges in the GTEx brain GCN were tested for sample label enrichment using the KINC.R package (https:/github.com/SystemsGenetics/ KINC.R). A Fisher's exact test with a Hochberg p-value correction was used as the default arguments to the analyzeNetCat function. The sample label enrichment lists for modules as well as edges are shown in Supplemental Table 2 and 3. In the sample label enrichment for modules, we considered to use the p-value threshold of $1 \mathrm{E}-3$ for significantly enriched modules. The edges with p-value less than $1 \mathrm{E}-10$ were considered as significantly enriched edges because this p-value is close to maximizing the number of edges and nodes. The number of enriched edges for each specific region with the adjusted p-value less than $1 \mathrm{E}-3,1 \mathrm{E}-5,1 \mathrm{E}-15$, and $1 \mathrm{E}-20$ were also collected separately and the tSNE visualization was run for each of those gene sets (Supplemental Table 4 and Supplemental Figure 1). Furthermore, we calculated the number of regions that each edge, module, and eQTL belonged to. eQTL datasets for 13 brain regions samples were also downloaded from the GTEx project $\mathrm{V7}^{10}$. region-specific edges and modules were selected to construct GTEx sub-brain GCNs. LCM modules were also tested for functional term enrichment using the FUNC-E package (https:/github.com/SystemsGenetics/ FUNC-E), which uses a Fisher's exact test similar to the David ${ }^{28}$ method of functional enrichment. For crossmodule comparisons, enriched terms were considered significant if the Bonferroni-corrected p-value was less than 0.001. Functional annotations performed include Gene Ontology ${ }^{29}$, Reactome ${ }^{30}, \mathrm{Pfam}^{31}$, Interpro ${ }^{32}$, and Mendelian Inheritance in Man (MIM) ${ }^{33}$. The full module functional enrichment list is shown in Supplemental Table 5. region-specific edges for each region also underwent functional term enrichment analysis, which is shown in Supplemental Table 6. Moreover, gene type for all currently identified genes was downloaded from Ensembl Biomart (https://useast.ensembl.org/info/data/biomart/). All genes from GTEx dataset, genes from brain GCN as well as each region-specific genes were counted for calculating the protein coding and non-coding gene percentages. This result is shown in Supplemental Table 7 and Supplemental Table 8.

t-SNE analysis. A dimensionality reduction and visualization pipeline was performed using either a full or partial GTEx brain GEM as the input. This allowed us to compare how varying subsets of genes were able to separate the selected brain regions. It was performed using the principal component analysis (PCA) and $\mathrm{t}$-distributed stochastic neighbor embedding (t-SNE) Python sklearn packages ${ }^{17}$. Each t-SNE run created a twodimensional randomly initialized embedding, in which samples were clustered into different sub-groups. The perplexity used for each run was 30. This pipeline was performed on the GTEx brain GCN GEM containing 1691 genes for 1671 samples, as well as the six GTEx sub-brain GCN GEMs containing region-specific genes for 1671 samples. We also performed PCA and t-SNE on the TCGA cancer GEM, which contained region-specific genes for 1431 tumor samples, in order to segregate the four TCGA tumor types. TCGA datasets were downloaded from TCGA data portal ${ }^{34}$.

Brian region classification. We used a two phase, bottom-up classification approach of a feedforward neural network, known as Gene Oracle ${ }^{15}$ (https://github.com/SystemsGenetics/gene-oracle), to classify brain regions, and thus, identify the region-specific gene biomarkers. Gene Oracle uses a multilayer perceptron (MLP) feedforward neural network ${ }^{35}$ to identify biomarker gene sets with a significant classification accuracy when comparing to sets with equal number of random genes. Gene Oracle can also sort genes within a gene set according to their classification rates. This is done by breaking the gene set down into its most discriminatory features, followed by iteratively appending genes to explore new combinations. The architecture of the network consists of a total of five layers: an input layer with a size equivalent to the size of the gene set, three hidden layers (512, 256, and 128 units, respectively), and a final layer for classification. The three hidden layers utilize rectified linear unit (ReLU) activation function ${ }^{36}$. In Gene Oracle phase I, six merged brain region gene sets were screened for a significant classification potential that would allow for classification of the samples into 13 brain regions. For each brain gene set, 50 random size-controlled gene sets were selected from all genes in the input GTEx GEM and evaluated using the same classifier. Size-control means that each corresponding gene in the random list was within $10 \%$ of the size of the original gene from the region-specific list. The mean classification accuracy was calculated for the 50 random gene sets and compared with the corresponding brain gene set accuracy. For example, the cortex set that contained 40 genes was compared to 50 different sets of 40 random size-controlled genes for classification accuracy. A 10-fold cross validation procedure was applied to train and test the model. A gene set was chosen to undergo further analysis if the classification accuracy was higher than that of the average of the corresponding random sets with a statistical significance of $\mathrm{p}<0.001$ (using Student's $t$ test). In Gene Oracle phase II, the gene set that exhibited a significant classification potential underwent a combinatorial decomposition in order to discover the most discriminatory genes in the set. Three brain gene sets with a smallest number of genes, including the cortex gene set, the spinal cord gene set, and the substantia nigra gene set, underwent Gene Oracle phase II to detect candidate genes for better classification.

To compare the results of Gene Oracle phase II, we utilized Random Forest ${ }^{37}$ to run the classification for the five brain sets that were significant in Phase I of Gene Oracle. Random Forest was also used to highlight the important features (i.e. genes) using its built in functions of scikit-learn library in Python. Once Random Forest identified the important features, they were compared to the ones identified by Gene Oracle. The Random Forest model consisted of 100 trees, where the value of the threshold for early stopping in tree growth is $1 \mathrm{E}-7$. The builtin scikit-learn function "RandomForestClassifier" was used to construct the Random Forest model in Python.

Tumor gene mutation rates. Somatic mutations for GBM, LGG, HNSC, PCPG, and KIRC tumor subtypes were downloaded from TCGA ${ }^{34}$. TCGA reported mutations from four different polymorphism detection methods including Muse ${ }^{38}$, Mutect ${ }^{39}$, Sniper $^{40}$ and Varscan ${ }^{41}$. We downloaded the Muse method dataset and counted the number of tumors with at least one mutation, the number of genes mutated in a tumor, and the 
number of total mutations present in a tumor. We summed these values for candidate gene sets and the sizecontrolled random gene sets of equal number. The randomized control genes were counted a hundred times and then an empirical $p$-value $(\mathrm{p}<0.01)$ was determined for each candidate gene set. The absence or presence of an IDH mutation (IDH1/IDH2/IDH3) in LGG and GBM samples was also collected and used for tSNE visualization.

Received: 4 February 2020; Accepted: 4 August 2020

Published online: 13 October 2020

\section{References}

1. Mahfouz, A., Huisman, S. M. H., Lelieveldt, B. P. F. \& Reinders, M. J. T. Brain transcriptome atlases: a computational perspective. Brain Struct. Funct. 222, 1557-1580. https://doi.org/10.1007/s00429-016-1338-2 (2017).

2. Richiardi, J. et al. Brain networks. Correlated gene expression supports synchronous activity in brain networks. Science 348, 11-14 (2015).

3. Lein, E. S. et al. Genome-wide atlas of gene expression in the adult mouse brain. Nature 445, 168-176. https://doi.org/10.1038/ nature05453 (2007).

4. Twine, N. A., Janitz, K., Wilkins, M. R. \& Janitz, M. Whole transcriptome sequencing reveals gene expression and splicing differences in brain regions affected by Alzheimer's disease. PLoS Onehttps://doi.org/10.1371/journal.pone.0016266 (2011).

5. Shen, E. H., Overly, C. C. \& Jones, A. R. The Allen Human Brain Atlas comprehensive gene expression mapping of the human brain. Trends Neurosci. 35, 711-714. https://doi.org/10.1016/j.tins.2012.09.005 (2010).

6. Negi, S. K. \& Guda, C. Global gene expression profiling of healthy human brain and its application in studying neurological disorders. Sci. Rep.https://doi.org/10.1038/s41598-017-00952-9 (2017).

7. Kirsch, L. \& Chechik, G. On expression patterns and developmental origin of human brain regions. PLoS Comput. Biol.https:// doi.org/10.1371/journal.pcbi.1005064 (2016).

8. Hawrylycz, M. et al. Canonical genetic signatures of the adult human brain. Nat. Neurosci.https://doi.org/10.1038/nn.4171 (2017).

9. Voineagu, I. Neurobiology of disease gene expression studies in autism : moving from the genome to the transcriptome and beyond. Neurobiol. Dis. 45, 69-75. https://doi.org/10.1016/j.nbd.2011.07.017 (2012).

10. Lonsdale, J. et al. The Genotype-Tissue Expression (GTEx) project. Nat. Genet. 45, 580-585 (2013). https://doi.org/10.1038/ng.2653. arXiv:NIHMS150003.

11. Zhang, B. \& Horvath, S. A general framework for weighted gene co-expression network analysis. Stat. Appl. Genet. Mol. Biol.https ://doi.org/10.2202/1544-6115.1128 (2005).

12. Ficklin, S. P. et al. Discovering condition-specific gene co-expression patterns using gaussian mixture models: a cancer case study. Sci. Rep.https://doi.org/10.1038/s41598-017-09094-4 (2017).

13. Ficklin, S. P. \& Feltus, F. A. A systems-genetics approach and data mining tool to assist in the discovery of genes underlying complex traits in Oryza sativa. Stat. Appl. Genet. Mol. Biol.https://doi.org/10.1371/journal.pone.0068551 (2013).

14. Gibson, S. M. et al. Massive-scale gene co-expression network construction and robustness testing using random matrix theory. PLoS Onehttps://doi.org/10.1371/journal.pone.0055871 (2013).

15. Targonski, C. A., Shearer, C. A., Shealy, B. T., Smith, M. C. \& Feltus, F. A. Uncovering biomarker genes with enriched classification potential from Hallmark gene sets. Sci. Rep.https://doi.org/10.1038/s41598-019-46059-1 (2019).

16. Ahn, Y. Y., Bagrow, J. P. \& Lehmann, S. Link communities reveal multiscale complexity in networks. Nature 466, 761-764 (2010). https://doi.org/10.1038/nature09182. arXiv:0903.3178.

17. van der Maaten, L. \& Hinton, G. Visualizing data using t-sne (2008).

18. Lee, J. H. \& Gleeson, J. G. The role of primary cilia in neuronal function. Neurobiol. Dis. 38, 167-172. https://doi.org/10.1016/j. nbd.2009.12.022 (2010).

19. Yan, H. et al. Idh1 and idh2 mutations in gliomas. N. Engl. J. Med. 360, 765-773 (2009).

20. Cao, S. et al. Dynamic host immune response in virus-associated cancers. Commun. Biol. 2, 109. https://doi.org/10.1038/s4200 3-019-0352-3 (2019).

21. Opocher, G. Genetics of pheochromocytomas and paragangliomas. Best Pract. Res. Clin. Endocrinol. Metab. 24, 943-956. https:// doi.org/10.1016/j.beem.2010.05.001 (2010).

22. Bolstad, B. preprocessCore: a collection of pre-processing functions (2018). R package version 1.42.0.

23. Dodge, Y. The concise encyclopedia of statistics (Springer Science and Business Media, New York, 2008).

24. Weinstein, J. N. et al. The cancer genome atlas pan-cancer analysis project. Nat. Genet. 45, 1113 (2013).

25. Roy, A., Pordes, R. \& Altunay, M. The open science grid.https://doi.org/10.1088/1742-6596/78/1/012057 (2007).

26. Poehlman, W. L., Rynge, M., Balamurugan, D., Mills, N. \& Feltus, F. A. Osg-kinc: High-throughput gene co-expression network construction using the open science grid. In 2017 IEEE International Conference on Bioinformatics and Biomedicine (BIBM), 1827-1831. https://doi.org/10.1109/BIBM.2017.8217938 (2017).

27. Deelman, E. et al. Pegasus, a workflow management system for science automation. Future Gen. Comput. Syst. 46, 17-35 (2015).

28. Huang, D. W. et al. The DAVID Gene Functional Classification Tool: a novel biological module-centric algorithm to functionally analyze large gene lists. Genome Biol.https://doi.org/10.1186/gb-2007-8-9-r183 (2007).

29. Carbon, S. et al. Expansion of the gene ontology knowledgebase and resources. Nucleic Acids Res. 45, D331-D338 (2017).

30. Fabregat, A. et al. The reactome pathway knowledgebase. Nucleic Acids Res. 44, 481-487. https://doi.org/10.1093/nar/gkv1351 (2016).

31. El-gebali, S. et al. The Pfam protein families database in 2019. Nucleic Acids Res. 47, 427-432. https://doi.org/10.1093/nar/gky99 5 (2019)

32. Mitchell, A. L. et al. InterPro in 2019: improving coverage, classification and access to protein sequence annotations. 47, 351-360, https://doi.org/10.1093/nar/gky1100 (2019).

33. Hamosh, A., Scott, A. F., Amberger, J., Valle, D. \& Mckusick, V. A. Online Mendelian Inheritance in Man (OMIM). Hum. Mutat. 61, 57-61 (2000)

34. Hoadley, K. A. et al. HHS Public Access. Cell 173, 291-304. https://doi.org/10.1016/j.cell.2018.03.022.Cell-of-Origin (2019).

35. Aitkin, M. \& Foxall, R. Statistical modelling of artificial neural networks using the multi-layer perceptron. Stat. Comput. 13, 227-239. https://doi.org/10.1023/A:1024218716736 (2003).

36. Nair, V. \& Hinton, G. E. Rectified linear units improve restricted Boltzmann machines. In Proceedings of the 27th International Conference on International Conference on Machine Learning, ICML'10, 807-814 (Omnipress, USA, 2010).

37. Liaw, A. \& Wiener, M. Classification and regression by randomforest. $R$ News 2, 18-22 (2002).

38. Fan, Y. et al. MuSE: accounting for tumor heterogeneity using a sample-specific error model improves sensitivity and specificity in mutation calling from sequencing data. Genome Biol.https://doi.org/10.1186/s13059-016-1029-6 (2016). 
39. Faria, Í. et al. Optimized pipeline of MuTect and GATK tools to improve the detection of somatic single nucleotide polymorphisms in whole- exome sequencing data. BMC Bioinform.https://doi.org/10.1186/s12859-016-1190-7 (2016).

40. Simola, D. F. \& Kim, J. Sniper: improved SNP discovery by multiply mapping deep sequenced reads. Genome Biol. 12, R55. https ://doi.org/10.1186/gb-2011-12-6-r55 (2011).

41. Koboldt, D. C. et al. VarScan: variant detection in massively parallel sequencing of individual and pooled samples. Bioinformatics 25, 2283-2285. https://doi.org/10.1093/bioinformatics/btp373 (2009).

\title{
Acknowledgements
}

The Genotype-Tissue Expression (GTEx) Project was supported by the Common Fund of the Office of the Director of the National Institutes of Health, and by NCI, NHGRI, NHLBI, NIDA, NIMH, and NINDS. The data used for the analyses described in this manuscript were obtained from the GTEx Portal GTEx Analysis v7. Clemson University is acknowledged for general computing on the Palmetto cluster. GCN construction was done using resources provided by the Open Science Grid, which is supported by the National Science Foundation award 1148698 , and the U.S. Department of Energy's Office of Science. FAF is supported by NSF projects \#1659300 and \#1444461.

\section{Author contributions}

Y.H. and F.A.F. conceived the experiment. Y.H. collected the datasets and wrote the manuscript. Y.H., M.A., B.H. and A.R.H. analyzed the data. W.L.P. contributed analysis tools. All of the authors have reviewed and approved the final manuscript.

\section{Competing interests}

The authors declare no competing interests.

\section{Additional information}

Supplementary information is available for this paper at https://doi.org/10.1038/s41598-020-73611-1.

Correspondence and requests for materials should be addressed to F.A.F.

Reprints and permissions information is available at www.nature.com/reprints.

Publisher's note Springer Nature remains neutral with regard to jurisdictional claims in published maps and institutional affiliations.

\begin{abstract}
(c) (i)
Open Access This article is licensed under a Creative Commons Attribution 4.0 International License, which permits use, sharing, adaptation, distribution and reproduction in any medium or format, as long as you give appropriate credit to the original author(s) and the source, provide a link to the Creative Commons licence, and indicate if changes were made. The images or other third party material in this article are included in the article's Creative Commons licence, unless indicated otherwise in a credit line to the material. If material is not included in the article's Creative Commons licence and your intended use is not permitted by statutory regulation or exceeds the permitted use, you will need to obtain permission directly from the copyright holder. To view a copy of this licence, visit http://creativecommons.org/licenses/by/4.0/.
\end{abstract}

(C) The Author(s) 2020 